BMJ Open

Diabetes

Research

\& Care

\title{
Correcting imbalance of sex hormones by a phosphodiesterase 5 inhibitor improves copulatory dysfunction in male rats with type 2 diabetes
}

\author{
Akiko Itoga (1) , Xinmin Zha, Keiko Nagase, Yoshitaka Aoki, Hideaki Ito, \\ Osamu Yokoyama
}

\begin{abstract}
To cite: Itoga A, Zha X, Nagase K, et al. Correcting imbalance of sex hormones by a phosphodiesterase 5 inhibitor improves copulatory dysfunction in male rats with type 2 diabetes. BMJ Open Diab Res Care 2020;8:e001111. doi:10.1136/ bmjdrc-2019-001111
\end{abstract}

Received 12 December 2019 Revised 19 March 2020 Accepted 24 March 2020
Check for updates

\section{(C) Author(s) (or their} employer(s)) 2020. Re-use permitted under CC BY-NC. No commercial re-use. See rights and permissions. Published by BMJ.

Department of Urology, Faculty of Medical Science, University of Fukui, Eiheiji-cho, Yoshidagun, Fukui, Japan

Correspondence to Akiko Itoga;

amohara@u-fukui.ac.jp

\section{ABSTRACT}

Introduction Sexual dysfunction is a common complication in men with type 2 diabetes and is often refractory to treatment. This study investigated the longterm influence of the phosphodiesterase 5 inhibitor (PDE5I) tadalafil on the level of sex hormones and sexual function in male Otsuka Long-Evans Tokushima Fatty (OLETF) rats as an animal model of spontaneous type 2 diabetes. Research design and methods We treated 36-weekold male OLETF and non-diabetic Long-Evans Tokushima Otsuka (LETO) rats with oral tadalafil $(100 \mu \mathrm{g} / \mathrm{kg} / \mathrm{day})$ for 12 weeks; sham groups received vehicle for 12 weeks. Before and after tadalafil treatment, serum levels of total and free testosterone, estradiol, luteinizing hormone (LH), follicle-stimulating hormone and proinflammatory cytokines were compared among four treatment groups. Copulatory function was examined by matching each rat to an estrous female. After completion of the experiment, total fat mass in the abdomen was measured.

Results Testosterone levels were significantly lower in OLETF versus LETO rats at 36 weeks. After 12 weeks of tadalafil treatment, levels of testosterone were significantly increased both in OLETF-tadalafil and LETO-tadalafil groups versus vehicle groups. Tadalafil decreased estradiol levels both in OLETF and LETO rats. Furthermore, tadalafil increased serum LH levels with a reduction of proinflammatory cytokines. Total fat mass was significantly lower in the OLETF-tadalafil group versus the OLETFvehicle group. A significant suppression of copulatory behavior, that is, elongation of intromission latency was found in OLETF rats. However, tadalafil treatment for 12 weeks shortened the intromission latency.

Conclusion Our results indicate that tadalafil treatment might improve copulatory disorder in the type 2 diabetic model via improvement of an imbalance in sex hormones and an increase in LH levels.

\section{INTRODUCTION}

Increasing evidence has pointed to a relationship between the presence of type 2 diabetes and sexual dysfunction in men, an effect that has been shown to reduce quality of life. ${ }^{12}$ The ratio of erectile dysfunction (ED) in patients with diabetes is 1.9 to 5 times than that of subjects without diabetes, and it is reported

\section{Significance of this study}

What is already known about this subject?

- Sexual dysfunction in men with type 2 diabetes is sometimes resistant to phosphodiesterase 5 inhibitors therapy.

- Phosphodiesterase 5 inhibitors were suggested to increase testosterone levels in patients with erectile dysfunction.

What are the new findings?

- Copulatory behavior was suppressed, that is, elongation of intromission latency, in rats with type 2 diabetes.

- Long-term treatment with phosphodiesterase 5 inhibitor tadalafil corrected sex hormone imbalances (increased testosterone and decreased estradiol levels), leading to improved copulatory disorder.

- Tadalafil treatment increased serum luteinizing hormone levels with the reduction of proinflammatory cytokines and decreased total fat mass in the abdomen.

How might these results change the focus of research or clinical practice?

- The present results should encourage research on correcting imbalance of sex hormones for improving sexual dysfunction such as copulatory disorder, especially in men with type 2 diabetes.

that $35 \%$ to $90 \%$ of male patients with type 2 diabetes suffer from sexual dysfunction, including ED and diminished sexual desire. ${ }^{34}$ The decrease in sexual desire in men with type 2 diabetes has been suggested to be caused by male hypogonadism. ${ }^{5}$

Phosphodiesterase 5 inhibitors (PDE5Is) such as sildenafil, tadalafil, and vardenafil have been recommended for first-line treatment of ED and are also widely used for the treatment of ED caused by diabetes. ${ }^{67}$ However, sexual dysfunction in men with type 2 diabetes is sometimes resistant to PDE5I therapy. ${ }^{8}$ Compared with placebo, tadalafil 
$2.5 \mathrm{mg}$ and $5 \mathrm{mg}$ taken once daily over 12 weeks has been reported to lead to a significant improvement in International Index of Erectile Function (IIEF) erectile function, intercourse satisfaction, and overall sexual satisfaction domains in patients with diabetes, but not in sexual desire domain. ${ }^{9}$ The success rate of PDE5I treatment in men with type 2 diabetes has been reported to be significantly lower when compared with men without diabetes. ${ }^{10}$ Tadalafil $5 \mathrm{mg}$ once daily was approved for the treatment of lower urinary tract symptoms suggestive of benign prostatic hyperplasia, and concomitant improvement of sexual function could be expected. ${ }^{11}{ }^{12}$ PDE5Is were also suggested to increase testosterone levels in patients with ED even with on-demand use. ${ }^{13} 14$ Therefore, long-term use of a PDE5I may improve sexual desire via elevated testosterone levels. To the best of our knowledge, no studies have been performed regarding the effects of long-term treatment with tadalafil on sexual dysfunction, especially sexual desire, in men with type 2 diabetes.

The Otsuka Long-Evans Tokushima Fatty (OLETF) rat is an animal model of human type 2 diabetes. ${ }^{15}$ Male OLETF rats fed normal chow show obesity, hyperlipidemia, hypertension and late onset of hyperglycemia (after 18 weeks of age), which resemble those of human type 2 diabetes. The control strain, non-diabetic LongEvans Tokushima Otsuka (LETO) rats is normal and never exhibits any components of diabetes. ${ }^{15}$ Although OLETF rats have ED with reduced level of serum testosterone, ${ }^{16}$ we expect that reduced testosterone will also result in copulatory behavioral disorders. In this study, we used OLETF rats as a model of type 2 diabetes and LETO rats as a control to investigate the effects of tadalafil on levels of testosterone, estradiol, luteinizing hormone (LH), follicle-stimulating hormone (FSH), and proinflammatory cytokines as well its effects on sexual function, especially copulatory behavior.

\section{MATERIALS AND METHODS}

\section{Animal preparation}

Four-week-old male LETO and OLETF rats and female Sprague-Dawley rats purchased from Japan SLC (Shizuoka, Japan) were housed at a constant temperature of $23^{\circ} \mathrm{C}$ and $50 \%$ to $60 \%$ humidity under a regular 12 hours light/dark schedule at the University of Fukui Animal Center. We treated 36-week-old male OLETF and LETO rats with oral tadalafil $(100 \mu \mathrm{g} / \mathrm{kg} /$ day $)$ for 12 weeks; the sham groups received only vehicle for 12 weeks. We divided into six groups: LETO-36w, LETO-ve-48w, LETO-ta-48w, OLETF-36w, OLETF-ve-48w, and OLETFta-48w (LETO-36w, 36-week-old LETO rats; LETOve-48w, 48-week-old LETO rats treated with vehicle for 12 weeks; LETO-ta-48w, 48-week-old LETO rats treated with tadalafil for 12 weeks; OLETF-36w, 36-week-old OLETF rats; OLETF-ve-48w, 48-week-old OLETF rats treated with vehicle for 12 weeks; OLETF-ta-48w, 48-week-old OLETF rats treated with tadalafil for 12 weeks). Each group has eight rats. Tap water and standard rat chow were freely available. All animal experiments were performed in accordance with the guidelines established by the Fukui University Committee for Animal Experimentation.

\section{Physiological and biochemical characteristics in LET0 and OLETF rats}

Mean body weight and systolic blood pressure (SBP) were monitored at the age of 36 and 48 weeks. SBP was measured in conscious rats with a computerized tailcuff system (BA-98A System; Softron, Tokyo, Japan) that determines SBP using a photoelectric sensor. Plasma

\begin{tabular}{|c|c|c|c|c|c|c|c|c|c|}
\hline $\begin{array}{l}\text { Weeks } \\
\text { of age }\end{array}$ & Strain & Tadalafil & $\mathbf{n}$ & Body weight (g) & $\begin{array}{l}\text { Systolic blood } \\
\text { pressure (mm } \\
\mathrm{Hg})\end{array}$ & $\begin{array}{l}\text { Plasma } \\
\text { glucose } \\
\text { (mg/dL) }\end{array}$ & $\begin{array}{l}\text { Plasma insulin } \\
(\mathrm{pg} / \mathrm{mL})\end{array}$ & $\begin{array}{l}\text { LDL- } \\
\text { cholesterol } \\
\text { (mg/dL) }\end{array}$ & $\begin{array}{l}\text { Plasma } \\
\text { triglyceride } \\
\text { (mg/dL) } \\
\end{array}$ \\
\hline \multirow[t]{2}{*}{36} & LETO & & 16 & $541.8 \pm 15.8$ & $201 \pm 4$ & $165 \pm 10$ & $5436 \pm 164$ & $13 \pm 1$ & $63 \pm 4$ \\
\hline & OLETF & & 16 & $661.5 \pm 18.4^{*}$ & $233 \pm 11^{*}$ & $199 \pm 7^{*}$ & $3713 \pm 153^{*}$ & $24 \pm 1^{*}$ & $179 \pm 10^{*}$ \\
\hline \multirow[t]{4}{*}{48} & LETO & Vehicle & 8 & $560.1 \pm 13.1$ & $215 \pm 8 \dagger$ & $180 \pm 3$ & $4778 \pm 279^{*}$ & $14 \pm 1$ & $69 \pm 2$ \\
\hline & & + & 8 & $544.2 \pm 16.8$ & $217 \pm 6 \dagger$ & $179 \pm 6$ & $4594 \pm 135^{\star}$ & $14 \pm 1$ & $68 \pm 3$ \\
\hline & OLETF & Vehicle & 8 & $649.8 \pm 21.7 \ddagger$ & $224 \pm 5$ & $224 \pm 10 \neq \S$ & $2906 \pm 124 \ddagger \S$ & $28 \pm 3 \neq 9$ & $247 \pm 17 \ddagger \S$ \\
\hline & & + & 8 & $633.1 \pm 13.8^{\star \star}$ & $216 \pm 10 \S$ & $215 \pm 12^{\star \star} \mathbb{q}$ & $2823 \pm 143 \S^{\star \star}$ & $18 \pm 1^{\star \star} \dagger \dagger$ & $214 \pm 9 \S^{\star \star} \dagger \dagger$ \\
\hline
\end{tabular}

${ }^{*} p<0.01$ versus LETO-36w.

$\dagger \mathrm{p}<0.05$ versus LETO-36w.

$\neq \mathrm{p}<0.01$ versus LETO-ve-48w.

$\S \mathrm{p}<0.01$ versus OLETF-36w.

Пp $<0.05$ vs OLETF-36w.

${ }^{* *} \mathrm{p}<0.01$ vs LETO-ta-48w.

$\dagger+\mathrm{p}<0.01$ versus OLETF-ve-48w.

LDL, low-density lipoprotein; LETO, Long-Evans Tokushima Otsuka; LETO-ta-48w, 48-week-old LETO rats treated with tadalafil for 12 weeks; ; LETO-ve-48w, 48-week-old LETO rats treated with vehicle for 12 weeks; LETO-36w, 36-week-old LETO rats; OLETF, Otsuka Long-Evans Tokushima Fatty; OLETF-ve-48w, 48-week-old OLETF rats treated with vehicle for 12 weeks; OLETF-36w, 36-week-old OLETF rats. 
glucose, triglycerides, cholesterol, and insulin levels were analyzed by SRL (Tokyo, Japan) at the age of 36 and 48 weeks.

\section{Multiplex cytokines and hormone analysis}

Free testosterone and total serum testosterone were measured at the age of 36 and 48 weeks by enzyme-linked immunoassay using the Rat Testosterone ELISA Kit and the Rat Free Testosterone ELISA Kit (Enzo Life Sciences International, New York, New York, USA) according to the manufacturer's instructions. Estradiol was measured by enzyme-linked immunoassay using the Estradiol ELISA Kit (Cayman Chemical, Ann Arbor, Michigan, USA) according to the manufacturer's instructions.

Serum concentrations of $\mathrm{LH}, \mathrm{FSH}$, interleukin- $1 \beta$ (IL-1 $\beta)$, IL-2, IL-4, IL-5, IL-6, IL-10, IL-13, interferon- $\gamma$ (IFN- $\gamma$ ), monocyte chemoattractant protein-1 (MCP-1), tumor necrosis factor- $\alpha$ (TNF- $\alpha$ ), and leptin of LETO and OLETF rats were evaluated using Milliplex MAP Kit (Millipore, Massachusetts, USA). Briefly, $25 \mu \mathrm{L}$ serum samples, standards, and quality controls were incubated with premixed antibody-immobilized beads for 2 hours in a shaker (TAITEC, Saitama, Japan). After washing, $25 \mu \mathrm{L}$ biotin-labeled detection antibodies were added and incubated with for 1 hour, followed by $25 \mu \mathrm{L}$ of streptavidinphycoerythrin incubation for $30 \mathrm{~min}$. After washing again, the magnetic beads were resuspended in $150 \mu \mathrm{L}$ of sheath fluid for $5 \mathrm{~min}$. Concentrations of cytokines and hormones in serum samples were measured using Luminex 200 instrument (Luminex, Austin, Texas, USA).

\section{Sexual behavior}

Female Sprague-Dawley rats were used as stimulus females for copulation tests. Each female rat was given estradiol benzoate intramuscularly at a daily dose of $1 \mathrm{mg}$ beginning 5 days before mating to induce estrus. A smear of vaginal mucus that was determined to be estrus was required for all female rats used in the study. We put estrus female rats into the cages of male rats one by one at 18:00 and recorded and evaluated the sexual activity for 12 hours at 36,42 , and 48 weeks of age. During the observation, the intromission latency, the mount frequency, and the intromission frequency were recorded. The intromission latency is the time from the introduction of the female until the first intromission. Prolonged latencies and
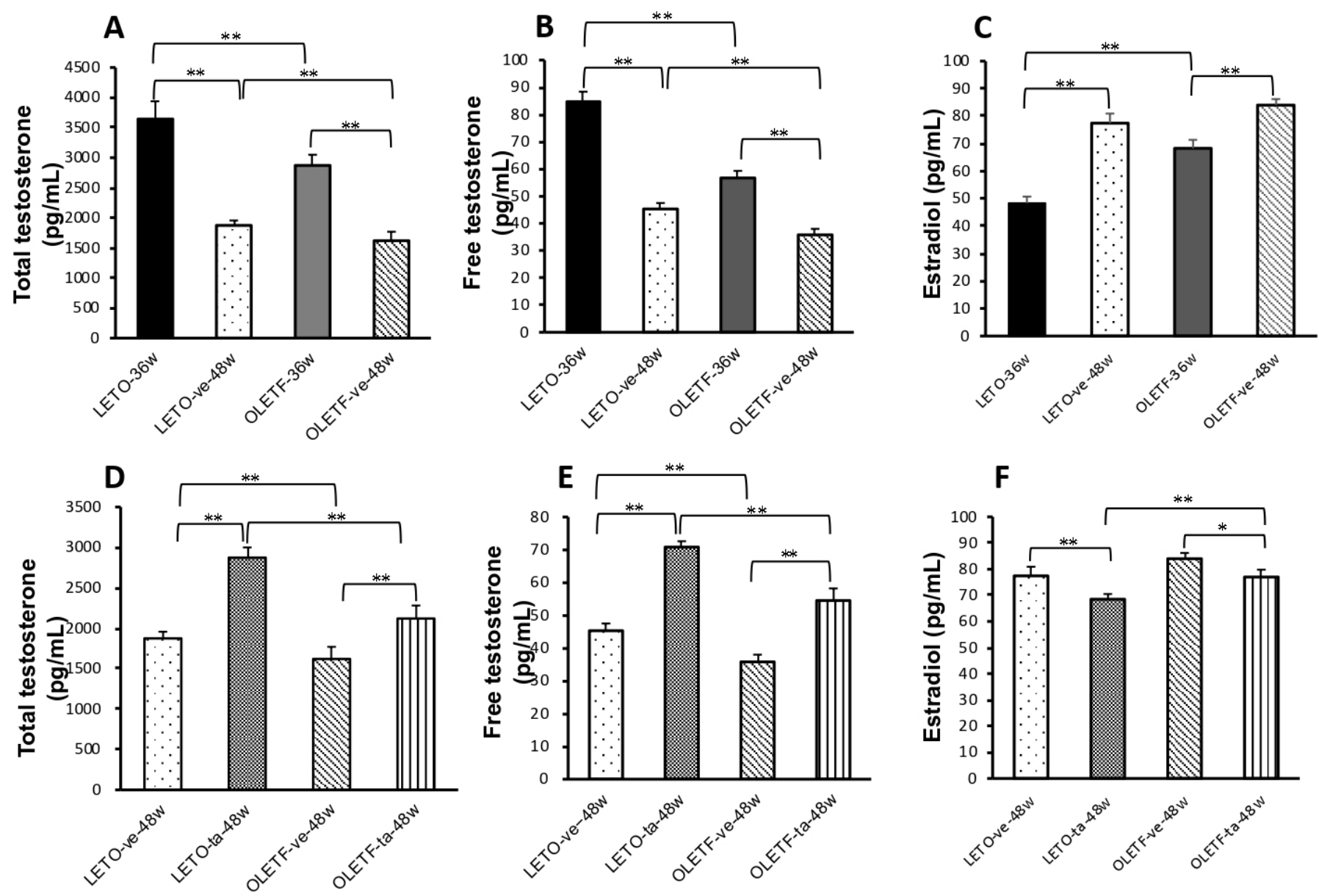

Figure 1 Changes in total testosterone $(A, D)$, free testosterone $(B, E)$, and estradiol $(C, F)$ in LETO and OLETF rats. * $p<0.05$, ${ }^{* *} p<0.01$ between values. LETO, Long-Evans Tokushima Otsuka; LETO-ta-48w; 48-week-old LETO rats treated with tadalafil for 12 weeks; LETO-ve-48w, 48-week-old LETO rats treated with vehicle for 12 weeks; LETO-36w, 36-week-old LETO rats; OLETF, Otsuka Long-Evans Tokushima Fatty; OLETF-ta-48w, 48-week-old OLETF rats treated with tadalafil for 12 weeks; OLETF-ve-48w, 48-week-old OLETF rats treated with vehicle for 12 weeks; OLETF-36w, 36-week-old OLETF rats. 

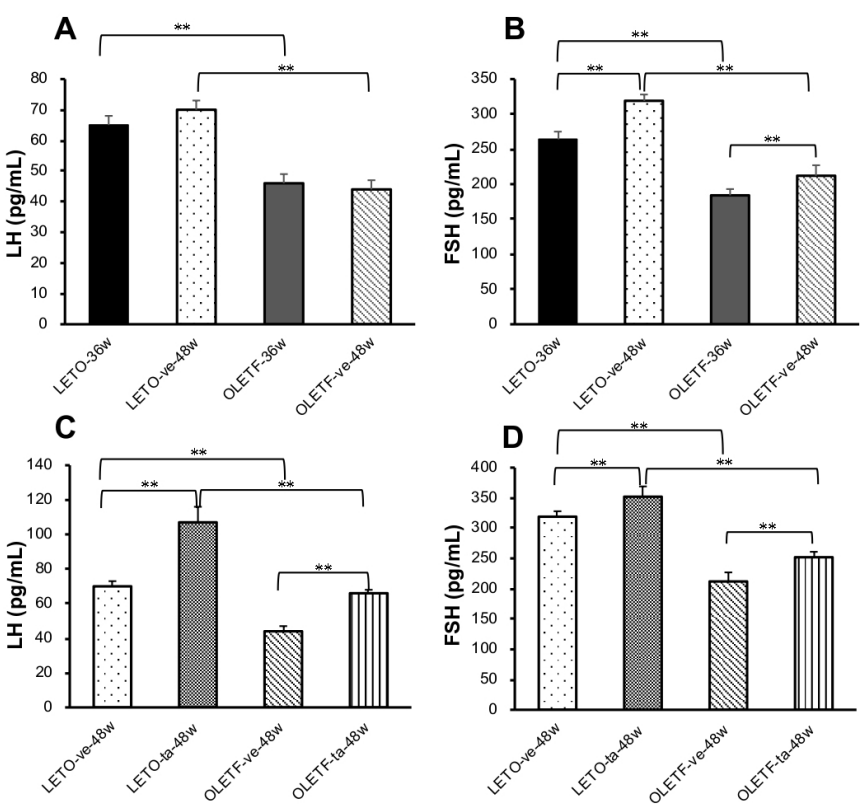

Figure 2 Changes in luteinizing hormone (LH) $(\mathrm{A}, \mathrm{C})$ and follicle-stimulating hormone (FSH) $(\mathrm{B}, \mathrm{D})$ in LETO and OLETF rats. ${ }^{* *} p<0.01$ between values. LETO, Long-Evans Tokushima Otsuka; LETO-ta-48w; 48-week-old LETO rats treated with tadalafil for 12 weeks; LETO-ve-48w, 48-week-old LETO rats treated with vehicle for 12 weeks; LETO-36w, 36-week-old LETO rats; OLETF, Otsuka Long-Evans Tokushima Fatty; OLETF-ta-48w, 48-week-old OLETF rats treated with tadalafil for 12 weeks; OLETF-ve-48w, 48-week-old OLETF rats treated with vehicle for 12 weeks; OLETF-36w, 36-week-old OLETF rats.

reduced frequency were indications of reduced sexual desire. $^{17}$

\section{Measurement of abdominal fat mass}

After completion of the experiment, rats were sacrificed with an overdose of pentobarbital $(60 \mathrm{mg} / \mathrm{kg})$. The abdomen of the rat was dissected and the weight of total fat mass in the abdomen was measured.

\section{Statistical analysis}

Results are presented as means \pm SEM. All data were analyzed by two-way analysis of variance using SPSS, V.16.0J for Windows. A $p$ value $<0.05$ was considered significant.

\section{RESULTS}

\section{Metabolic analysis of LETO and OLETF rats}

Body weight, plasma glucose levels, low-density lipoprotein (LDL)-cholesterol, and triglycerides were significantly higher in OLETF rats versus LETO rats at 36 weeks of age, and in OLETF rats versus LETO rats at 48 weeks (vehicle treated) of age (table 1 ). Treatment with tadalafil for 12 weeks significantly decreased plasma LDLcholesterol and triglyceride levels in OLETF rats, but not in LETO rats.

\section{Hormonal analysis}

Total and free testosterone levels were significantly lower in LETO and OLETF rats at 48 weeks vs 36 weeks of age (figure 1A,B). However, estradiol levels were significantly higher in LETO and OLETF rats at 48 weeks vs 36 weeks of age (figure 1C). Twelve-week treatment with tadalafil increased total and free testosterone levels and decreased estradiol levels in both rat types (figure 1D-F). LH levels were significantly higher in LETO rats at 48 weeks compared with 36 weeks (figure 2C). In contrast, no significant differences in LH levels were seen in OLETF rats between these time points (figure 2A). FSH levels were significantly higher in LETO and OLETF rats at 48 weeks compared with 36 weeks irrespective of tadalafil treatment (figure 2B,D). Twelve-week treatment with tadalafil increased LH levels in OLETF rats $(p<0.01)$ (figure 2C).

\section{Proinflammatory cytokine analysis}

TNF- $\alpha$ levels were significantly higher in OLETF rats than those in LETO rats at 36 weeks of age (figure 3A). TNF- $\alpha$ levels of LETO rats did not change from 36 weeks compared with 48 weeks; however, levels in OLETF rats significantly increased over this time period. Twelve-week treatment with tadalafil significantly decreased the TNF- $\alpha$ levels of OLETF rats (figure 3B). Levels of other cytokines such as IL-1 $\beta$, IL-2, IL-6, MCP-1, IFN- $\gamma$, and leptin also increased with age and diabetes. However, levels of these cytokines decreased with 12-week treatment with tadalafil (data not shown). Levels of IL-4, IL-10 and IL-13 showed the opposite responses, that is, they decreased with age and diabetes and increased with 12-week treatment with tadalafil (data not shown).
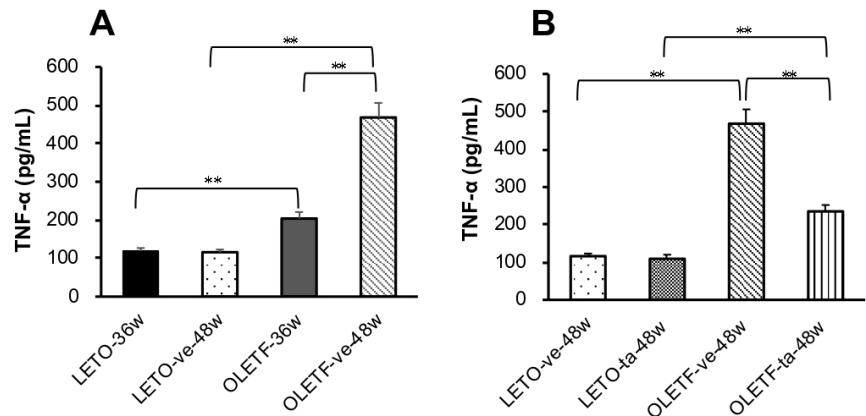

Figure 3 Changes in tumor necrosis factor- $\alpha$ (TNF- $\alpha$ ) after 12-week treatment with vehicle or tadalafil in LETO and OLETF rats at 36 weeks to 48 weeks of age $(A)$ and at 48 weeks of age (B). ${ }^{* *} p<0.01$ between values. LETO, Long-Evans Tokushima Otsuka; LETO-ta-48w; 48-week-old LETO rats treated with tadalafil for 12 weeks; LETO-ve-48w, 48-week-old LETO rats treated with vehicle for 12 weeks; LETO-36w, 36-week-old LETO rats; OLETF, Otsuka LongEvans Tokushima Fatty; OLETF-ta-48w, 48-week-old OLETF rats treated with tadalafil for 12 weeks; OLETF-ve-48w, 48-week-old OLETF rats treated with vehicle for 12 weeks; OLETF-36w, 36-week-old OLETF rats. 

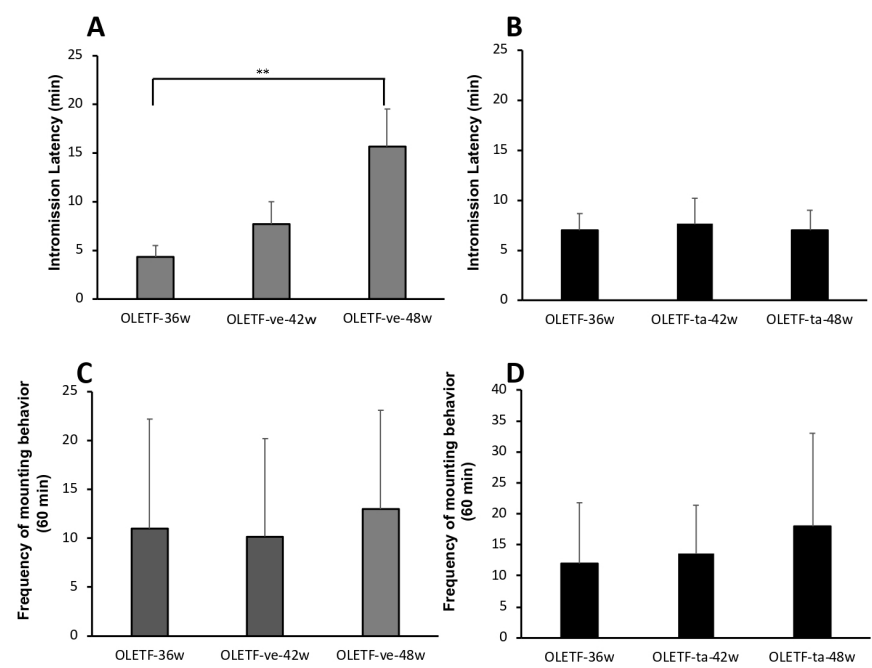

Figure 4 Changes in intromission latency $(A, B)$ and frequency of mounting behavior $(C, D)$ for 1 hour before and after 6 or 12 weeks treatment with vehicle $(A, C)$ or tadalafil $(B, D)$ in OLETF rats. ${ }^{* \star} p<0.01$ between values. OLETF, Otsuka Long-Evans Tokushima Fatty; OLETF-ta-42w, 42-week-old OLETF rats treated with tadalafil for 6 weeks; OLETF-ta-48w, 48-week-old OLETF rats treated with tadalafil for 12 weeks; OLETF-ve-42w, 42-week-old OLETF rats treated with vehicle for 6 weeks; OLETF-ve-48w, 48-weekold OLETF rats treated with vehicle for 12 weeks; OLETF36 w, 36-week-old OLETF rats.

\section{Sexual behavior}

Intromission latency was significantly higher in OLETF versus LETO rats at 36 weeks of age (LETO-36w: $4.0 \pm 0.4 \mathrm{~min}$; OLETF-36w: $8.1 \pm 0.7 \mathrm{~min} ; \mathrm{p}<0.05)$. Intromission latency increased from 36 weeks to 42 weeks, and 48 weeks of age in OLETF rats treated with vehicle (figure 4A). Significant increase in intromission latency was found between 36 and 48 weeks of age $(p<0.01)$. This finding represents suppression of mounting behavior. No significant change in intromission latency was found in LETO rats (LETO-ve-48w: $6.75 \pm 2.6 \mathrm{~min}$ ).

Intromission latency did not change from 36 to 48 weeks of age in OLETF rats treated with tadalafil (figure 4B). Twelve-week treatment with tadalafil prevented mounting ability from worsening. No change in frequency of mounting behavior was found after 12 weeks of treatment with vehicle or tadalafil (figure 4C,D).

\section{Abdominal fat mass}

LETO rats showed no increase in visceral fat weight at 48 weeks compared with 36 weeks of age (figure 5A). In contrast, OLETF rats showed a significant increase in visceral fat weight between 36 and 48 weeks of age $(p<0.01)$ (figure 5B). Twelve-week treatment with tadalafil significantly decreased visceral fat weight in both rats (both, $\mathrm{p}<0.01$ ).

\section{DISCUSSION}

OLETF rats fed normal chow showed obesity, hyperlipidemia, hypertension, and hyperglycemia with
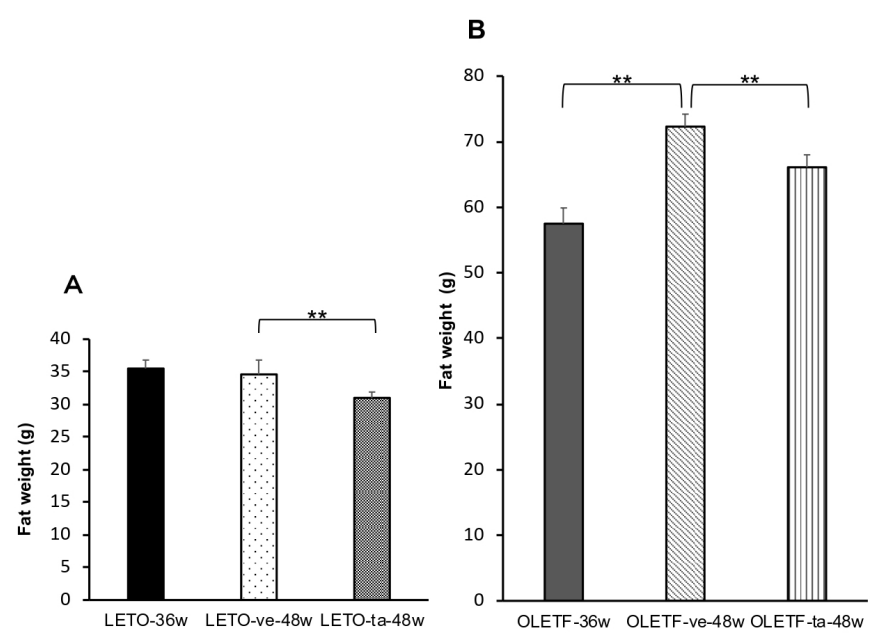

Figure 5 Changes in visceral fat weight in LETO $(A)$ and OLETF (B) rats. ${ }^{* *} p<0.01$ between values. LETO, Long-Evans Tokushima Otsuka; LETO-ta-48w; 48-week-old LETO rats treated with tadalafil for 12 weeks; LETO-ve-48w, 48-weekold LETO rats treated with vehicle for 12 weeks; LETO36w, 36-week-old LETO rats; OLETF, Otsuka Long-Evans Tokushima Fatty; OLETF-ta-48w, 48-week-old OLETF rats treated with tadalafil for 12 weeks; OLETF-ve-48w, 48-weekold OLETF rats treated with vehicle for 12 weeks; OLETF36w, 36-week-old OLETF rats.

hypogonadism, which caused copulatory disorder. Oral administration of tadalafil for 12 weeks corrected sex hormone imbalances (increased testosterone and decreased estradiol levels) in OLETF rats, leading to improved copulatory disorder. Tadalafil treatment increased serum LH levels with the reduction of proinflammatory cytokines and decreased total fat mass in the abdomen. This study is the first to reveal the effects of PDE5I on copulatory disorder in rats with type 2 diabetes.

Epidemiological studies of men with type 2 diabetes have shown that hypogonadism is associated with sexual dysfunction, including ED and diminished sexual desire. $^{18} 19$ Pathological animal models resembling hormonal dynamics of humans are necessary for pathological clarification and optimal treatment of sexual dysfunction in men with type 2 diabetes. OLETF rats are considered to be a model close to the natural history of type 2 diabetes in humans and have hyperinsulinemia and hypertension associated with obesity, and eventually changes from weight loss to low insulin. ${ }^{15} \mathrm{ED}$ and copulatory dysfunction also appeared in the present study. Given this series of courses, it is considered to be suitable as a model for long-term administration of tadalafil. One study showed that testis weight decreased in OLETF rats compared with LETO rats, but no differences were found in testosterone or estradiol levels measured by a dextrancharcoal assay. ${ }^{20}$ In contrast, bioavailable testosterone, which was measured by liquid chromatography-tandem mass spectrometry method, has been shown to be significantly lower in OLETF rats at 20 weeks of age compared with LETO rats. ${ }^{16}$ In the present study, total and free testosterone levels were significantly lower in OLETF rats 
when compared with LETO rats at 36 weeks of age. These levels were measured by rat testosterone ELISA Kit and rat free testosterone ELISA kit. Therefore, it is speculated that differences in testosterone levels among rats of the same strain are due to different measurement methods.

Five-week testosterone replacement therapy (TRT) has been suggested to be effective for ED in OLETF rats by improving endothelial function and inflammation. ${ }^{16}$ TRT is widely used in patients with symptoms of hypogonadism, although it is not commonly used as a preventive intervention or for treatment for male patients with type 2 diabetes. There are some controversies regarding the use of TRT for hypogonadism associated with type 2 diabetes. ${ }^{21}{ }^{22}$ TRT may cause side effects such as benign prostatic hyperplasia or cancer, cardiovascular risk due to increased red blood cells, and infertility. TRT should be used carefully with regular monitoring.

TRT was ineffective in improving ED in situations where serum levels of estradiol were high. ${ }^{23}$ Estradiol induces ED by enhancing Rho-kinase-mediated smooth muscle contraction. Therefore, increases in serum testosterone levels have the potential to improve erectile functionof male patients with diabetes as long as serum estradiol levels are kept low. After the 12 weeks of tadalafil treatment in this study, levels of total and free testosterone increased both in OLETF and LETO rats. Furthermore, tadalafil treatment decreased levels of estradiol. The shortened intromission latency as a sign of copulatory function in this study was likely the result of the improvement in the imbalance between serum testosterone and estradiol. There are some reports that ED occurs when the balance between testosterone and estradiol is lost. ${ }^{23}$ However, no reports could be found on sexual behavior such as copulatory function.

Sexual dysfunction includes erectile, ejaculatory, and copulatory dysfunction. There are many reports that intromission latency, mount frequency are useful as parameters of copulatory function. Social stress has been reported to induce marked suppression of copulatory behavior, for example, elongation of intromission in male rats. ${ }^{17}$ Transplantation of interstitial cell suspension containing hormone-producing cells facilitates a restoration of copulatory activity, resulting in reduced intromission latency. ${ }^{24}$ In this study, the intromission latency was significantly higher in OLETF rats than in LETO rats, indicating that OLETF rats had copulatory dysfunction. Male copulatory behavior is based on sexual desire (libido), which depends on the presence of testosterone. ${ }^{25}{ }^{26} \mathrm{~A}$ decrease in serum testosterone levels is thought to cause copulatory dysfunction. Clinically, on-demand administration of flexible dosages of tadalafil $(5,10$, and $20 \mathrm{mg})$ in men has been reported to induce significant decreases in serum estradiol levels with a significant increase in the testosterone/estradiol ratio, although total and free testosterone did not change. ${ }^{26}$ Furthermore, 3 -month treatment with tadalafil $5 \mathrm{mg}$ once daily increased serum testosterone levels and was effective for ED in men caused by low testosterone levels due to metabolic syndrome. ${ }^{27}$ Therefore, tadalafil itself does not improve the copulatory disorder or increase sexual desire, but rather increases testosterone levels, which leads to improved copulatory function in the present study. As a result, it is possible that tadalafil treatment may prevent sexual activity from worsening in men with type 2 diabetes.

Several findings may explain why tadalafil treatment increases testosterone levels. Immunohistochemistry and western blotting analysis confirmed that PDE5I is localized in Leydig and peritubular cells of the rat testis, as well as in vascular smooth muscle cells. ${ }^{28}$ Chronic treatment with sildenafil for 1 month increased cyclic guanosine monophosphate and promoted cholesterol uptake into the mitochondria of Leydig cells in male Swiss Webster mice. The accumulation of cyclic guanosine monophosphate could be involved in the stimulation of androgen biosynthesis in Leydig cells. ${ }^{29}$

In the present study, increases in serum testosterone levels with tadalafil were associated with decreases in serum estradiol levels and total fat mass. Another study showed that treatment with PDE5I sildenafil $100 \mathrm{mg} /$ day for 12 weeks reduced waist circumference and improved visceral adiposity in male patients with type 2 diabetes. ${ }^{30} \mathrm{~A}$ recent report indicated that acute exposure to PDE5Is induced aromatase expression with an increase in estradiol concentrations in human visceral adipocytes. ${ }^{31}$ However, this observation does not explain the decrease in circulating estradiol levels in men with ED treated with long-term tadalafil. ${ }^{32}$ Using human osteoblast-like cells, it has been shown that tadalafil inhibits mRNA and protein expression of aromatase, which results in increases in testosterone levels in the supernatants. ${ }^{33}$ Furthermore, tadalafil increased protein expression of androgen receptors and decreased estrogen receptors. ${ }^{33}$ Long-term treatment with tadalafil might modulate aromatase activity, and this activity might be dependent on the target organs. Twelve-week treatment with tadalafil decreased the accumulation of visceral adipose tissue and improved hypertriglyceridemia in the rabbit by restoring insulin sensitivity and promoting differentiation of preadipocytes. ${ }^{34}$ Furthermore, tadalafil has been shown to induce the expression of the brown fat-specific marker uncoupling protein 1 in visceral adipose tissue, leading to the conversion of white adipose tissue to brown adipose tissue. ${ }^{34}$ The aromatase cytochrome $\mathrm{P} 450$ enzyme, which is responsible for a key step in the biosynthesis of estradiol, is highly expressed in white adipose tissue. ${ }^{35}$ It is surmised that tadalafil decreases aromatase activity by changing white adipose tissue to brown adipose tissue.

A positive relationship was suggested between chronic inflammation or a proinflammatory state and obesity in animal models. ${ }^{36}{ }^{37}$ White adipose tissue produces a large number of inflammatory factors including proinflammatory cytokines, such as IL-1 $\beta$, IL- 6 , and TNF- $\alpha$, which are associated with insulin resistance. ${ }^{37}$ In contrast, reducing inflammation in obese subjects has been shown to improve insulin resistance. ${ }^{38}$ Furthermore, 
proinflammatory cytokines have been found to influence the hypothalamic-pituitary-gonadal axis. ${ }^{39}$ An increase in circulating TNF- $\alpha$ levels directly inhibits LH excretion from the pituitary gland, which results in decreased serum testosterone levels. ${ }^{40}$ As shown in the present study, 12-week treatment with tadalafil slightly improved glucose intolerance and increased serum testosterone and LH levels with a reduction of proinflammatory cytokines. In diabetic mice, 1-month treatment with tadalafil also reduced circulating proinflammatory cytokines such as TNF- $\alpha$ and IL-1 $\beta{ }^{41}$ Therefore, long-term treatment with tadalafil may improve sexual activity in patients with type 2 diabetes through its effects on the gonadal organs, adipose tissue, and pituitary gland. The increase in testosterone levels in the present study may be due to inhibitory effects of tadalafil on proinflammatory cytokines and resulting increases in $\mathrm{LH}$, in addition to its direct effect on the testis. The lifespan of a rat is about 96 weeks. The dosing period corresponds to one-eighth, 10 years of a human life (80 years). Therefore, it corresponds to administration from the age of 40 to 50 years. Tadalafil requires long-term administration to improve copulatory disorders in patients with type 2 diabetes. Further investigations are necessary to explore the target organ of PDE5I related to increases in testosterone levels.

\section{Limitations}

This study has several limitations. First, measurement of the aromatase activity of the adipose tissue could not be carried out. Therefore, the cause of the decrease in estradiol levels could not be identified. Second, it is an animal study and results may not apply to humans.

\section{CONCLUSIONS}

In conclusion, type 2 diabetes might induce an imbalance of sex hormones, resulting in sexual dysfunction such as copulatory disorder. Treatment with the PDE5I tadalafil for 12 weeks was associated with an increased testosterone/estradiol ratio and improved copulatory function. Tadalafil also has the potential to increase serum LH levels, reduce proinflammatory cytokines, and decrease total abdominal fat mass.

\section{Acknowledgements The authors thank all the project members.}

Contributors Al: planning, animal experiments, data collection, and paper writing. $\mathrm{XZ}$ : animal experiments. $\mathrm{KN}$ : animal experiments. YA: data analysis. HI: data collection. OY: planning and paper writing.

Funding The study was conducted with financial support from Nippon Shinyaku.

Competing interests OY has received consultancy, lectureship, and advisory board membership fees and editorial assistance from Astellas Pharma; consultancy, lectureship, and advisory board membership fees from Kyorin and Kissei; lectureship fees from Pfizer and Nippon Shinyaku; and consultancy fees from GSK and Taiho.

\section{Patient consent for publication Not required.}

Ethics approval This study was approved by the Institutional Animal Care and Use Committee (Permission no: R01056) and carried out according to the guidelines established by the Fukui University Committee for Animal Experimentation.

Provenance and peer review Not commissioned; externally peer reviewed.
Data availability statement No data are available.

Open access This is an open access article distributed in accordance with the Creative Commons Attribution Non Commercial (CC BY-NC 4.0) license, which permits others to distribute, remix, adapt, build upon this work non-commercially, and license their derivative works on different terms, provided the original work is properly cited, appropriate credit is given, any changes made indicated, and the use is non-commercial. See: http://creativecommons.org/licenses/by-nc/4.0/.

ORCID iD

Akiko Itoga http://orcid.org/0000-0002-9493-663X

\section{REFERENCES}

1 Malavige LS, Jayaratne SD, Kathriarachchi ST, et al. Erectile dysfunction among men with diabetes is strongly associated with premature ejaculation and reduced libido. J Sex Med 2008;5:2125-34.

2 De Berardis G, Franciosi M, Belfiglio M, et al. Erectile dysfunction and quality of life in type 2 diabetic patients: a serious problem too often overlooked. Diabetes Care 2002;25:284-91.

3 Hatzimouratidis K, Amar E, Eardley I, et al. Guidelines on male sexual dysfunction: erectile dysfunction and premature ejaculation. Eur Urol 2010:57:804-14.

4 Habibi A, Kalbasi S, Saadatjoo SA, et al. Evaluation of erectile dysfunction and associated factors in type-II diabetic patients in Birjand, Iran in 2008-2009. J Res Health Sci 2011;11:97-102.

5 Fushimi $\mathrm{H}$, Horie $\mathrm{H}$, Inoue $\mathrm{T}$, et al. Low testosterone levels in diabetic men and animals: a possible role in testicular impotence. Diabetes Res Clin Pract 1989;6:297-301.

6 Montague DK, Jarow JP, Broderick GA, et al. For the erectile dysfunction guideline update panel. Chapter 1: the management of erectile dysfunction: an AUA update. J Urol 2005;174:230-9.

7 Bruzziches R, Greco EA, Pili M, et al. Redefining the role of longacting phosphodiesterase inhibitor tadalafil in the treatment of diabetic erectile dysfunction. Curr Diabetes Rev 2008;4:24-30.

8 Buvat J, van Ahlen $\mathrm{H}$, Schmitt $\mathrm{H}$, et al. Efficacy and safety of two dosing regimens of tadalafil and patterns of sexual activity in men with diabetes mellitus and erectile dysfunction: scheduled use vs. on-demand regimen evaluation (sure) study in 14 European countries. J Sex Med 2006;3:512-20.

9 Hatzichristou D, Gambla M, Rubio-Aurioles E, et al. Efficacy of tadalafil once daily in men with diabetes mellitus and erectile dysfunction. Diabet Med 2008;25:138-46.

10 Boulton AJ, Selam JL, Sweeney M, et al. Sildenafil citrate for the treatment of erectile dysfunction in men with type II diabetes mellitus. Diabetologia 2001;44:1296-301.

11 Yokoyama O, Yoshida M, Kim SC, et al. Tadalafil once daily for lower urinary tract symptoms suggestive of benign prostatic hyperplasia: a randomized placebo- and tamsulosin-controlled 12-week study in Asian men. Int J Urol 2013;20:193-201.

12 Giuliano F, Oelke M, Jungwirth A, et al. Tadalafil once daily improves ejaculatory function, erectile function, and sexual satisfaction in men with lower urinary tract symptoms suggestive of benign prostatic hyperplasia and erectile dysfunction: results from a randomized, placebo- and tamsulosin-controlled, 12-week double-blind study. $J$ Sex Med 2013;10:857-65.

13 Carosa E, Martini P, Brandetti F, et al. Type V phosphodiesterase inhibitor treatments for erectile dysfunction increase testosterone levels. Clin Endocrinol 2004;61:382-6.

14 Yasuda M, Ide H, Furuya K, et al. Salivary 8-OHdG: a useful biomarker for predicting severe ED and hypogonadism. J Sex Med 2008;5:1482-91.

15 Kawano K, Hirashima T, Mori S, et al. Spontaneous long-term hyperglycemic rat with diabetic complications. Otsuka Long-Evans Tokushima fatty (OLETF) strain. Diabetes 1992;41:1422-8.

16 Kataoka T, Hotta Y, Maeda Y, et al. Assessment of androgen replacement therapy for erectile function in rats with type 2 diabetes mellitus by examining nitric oxide-related and inflammatory factors. J Sex Med 2014;11:920-9.

17 Mizuno T, Yotsuyanagi S, Nagasaka Y, et al. Dehydroepiandrosterone alleviates copulatory disorder induced by social stress in male rats. $J$ Sex Med 2006;3:612-8.

18 Ding EL, Song Y, Malik VS, et al. Sex differences of endogenous sex hormones and risk of type 2 diabetes: a systematic review and meta-analysis. JAMA 2006;295:1288-99.

19 Phé V, Rouprêt M. Erectile dysfunction and diabetes: a review of the current evidence-based medicine and a synthesis of the main available therapies. Diabetes Metab 2012;38:1-13. 
20 Komaki K, Ohno Y, Aoki N. Gonadal hormones and gonadal function in type 2 diabetes model OLETF (Otsuka long Evans Tokushima fatty) rats. Endocr J 2005;52:345-51.

21 Fink J, Matsumoto M, Tamura Y. Potential application of testosterone replacement therapy as treatment for obesity and type 2 diabetes in men. Steroids 2018;138:161-6.

22 Hassanabad MF, Fatehi M. Androgen therapy in male patients suffering from type 2 diabetes: a review of benefits and risks. Curr Diabetes Rev 2018.

23 Kataoka T, Hotta Y, Ohno M, et al. Limited effect of testosterone treatment for erectile dysfunction caused by high-estrogen levels in rats. Int J Impot Res 2013;25:201-5.

24 Deng B, Bondarenko T, Pakhomov O. Changes in sexual behavior of orchidectomized rats under influence of allotransplantation of testicular interstitial cell suspension. Cell Transplant 2017;26:795-803.

25 Baum MJ, Vreeburg JT. Copulation in castrated male rats following combined treatment with estradiol and dihydrotestosterone. Science 1973;182:283-5

26 Niikura S, Yokoyama O, Komatsu K, et al. A causative factor of copulatory disorder in rats following social stress. J Urol 2002;168:843-9.

27 Ozcan L, Polat EC, Kocaaslan R, et al. Effects of taking tadalafil 5 mg once daily on erectile function and total testosterone levels in patients with metabolic syndrome. Andrologia 2017;49:12751.

28 Scipioni A, Stefanini S, Santone R, et al. Immunohistochemical localisation of PDE5 in Leydig and myoid cells of prepuberal and adult rat testis. Histochem Cell Biol 2005;124:401-7.

29 Saraiva KLA, Silva AKSE, Wanderley MI, et al. Chronic treatment with sildenafil stimulates Leydig cell and testosterone secretion. Int J Exp Pathol 2009;90:454-62.

30 Fiore D, Gianfrilli D, Giannetta E, et al. Pde5 inhibition ameliorates visceral adiposity targeting the miR-22/SIRT1 pathway: evidence from the CECSID trial. J Clin Endocrinol Metab 2016;101:1525-34.
31 Aversa A, Caprio M, Antelmi A, et al. Exposure to phosphodiesterase type 5 inhibitors stimulates aromatase expression in human adipocytes in vitro. J Sex Med 2011;8:696-704.

32 Greco EA, Pili M, Bruzziches R, et al. Testosterone:estradiol ratio changes associated with long-term tadalafil administration: a pilot study. J Sex Med 2006;3:716-22.

33 Aversa A, Fittipaldi S, Bimonte VM, et al. Tadalafil modulates aromatase activity and androgen receptor expression in a human osteoblastic cell in vitro model. J Endocrinol Invest 2016;39:199-205.

34 Maneschi E, Cellai I, Aversa A, et al. Tadalafil reduces visceral adipose tissue accumulation by promoting preadipocytes differentiation towards a metabolically healthy phenotype: studies in rabbits. Mol Cell Endocrinol 2016;424:50-70.

35 Cabler S, Agarwal A, Flint M, et al. Obesity: modern man's fertility nemesis. Asian J Androl 2010;12:480-9.

36 Divella R, De Luca R, Abbate I, et al. Obesity and cancer: the role of adipose tissue and adipo-cytokines-induced chronic inflammation. $J$ Cancer 2016;7:2346-59.

37 Griffin C, Lanzetta N, Eter L, et al. Sexually dimorphic myeloid inflammatory and metabolic responses to diet-induced obesity. Am J Physiol Regul Integr Comp Physiol 2016;311:R211-6.

38 Weisberg SP, Hunter D, Huber R, et al. Ccr2 modulates inflammatory and metabolic effects of high-fat feeding. J Clin Invest 2006;116:115-24.

39 Tsatsanis C, Dermitzaki E, Avgoustinaki P, et al. The impact of adipose tissue-derived factors on the hypothalamic-pituitarygonadal (hpg) axis. Hormones 2015;14:549-62.

40 Iwasa T, Matsuzaki T, Murakami M, et al. Neonatal lipopolysaccharide exposure attenuates the homotypic stressinduced suppression of LH secretion in adulthood in male rat. Int $J$ Dev Neurosci 2009;27:345-9.

41 Varma A, Das A, Hoke NN, et al. Anti-Inflammatory and cardioprotective effects of tadalafil in diabetic mice. PLoS One 2012;7:e45243. 